\title{
Instantáneas
}

\section{Aplicación de la técnica de respuestas aleatorias para analizar la prevalencia de abortos provocados en México}

El aborto provocado está legalmente restringido en México y constituye un estigma social grave. Muchas mexicanas con embarazos no deseados se someten a abortos ilegales, una gran parte de ellos realizados en condiciones antihigiénicas. En esta situación, es difícil obtener información confiable acerca de la frecuencia de abortos provocados. Según diferentes fuentes, en México se realizan entre 100000 y 500000 abortos provocados anualmente.

En este estudio se estimó el número de abortos provocados mediante la técnica de respuestas aleatorias (TRA) en una muestra aleatoria multietápica de 1792 mujeres mexicanas de 15 a 55 años de edad. Además, se analizan las características de las mujeres que informaron haberse sometido a un aborto y mediante un modelo de regresión logística múltiple se identifican los factores asociados significativamente con este hecho. En el artículo se analiza ampliamente la factibilidad y aceptación de la técnica de investigación aplicada.

La TRA, desarrollada en la década de 1960, es una técnica de recopilación de información que protege la privacidad de los encuestados sobre temas considerados sensibles. Para ello se combinan las preguntas sensibles con otras no sensibles cuyas respuestas probables son "sí". El entrevistador no sabe la naturaleza de la respuesta captada. Finalmente se estima la proporción de personas que informó haber tenido una conducta o haber realizado un acto censurable según: a) la probabilidad de seleccionar una pregunta considerada sensible; b) la frecuencia de respuestas afirmativas a preguntas no sensibles; y c) el número de participantes que respondió "sí" a alguna pregunta, ya sea sensible o no sensible.

Los resultados de la aplicación de la TRA a esta muestra nacional demostró que la mayoría de las mexicanas $(52,1 \%)$ consideró que el aborto provocado debía prohibirse independientemente de las circunstancias, mientras $41,9 \%$ declaró que este tipo de aborto debía permitirse en determinados casos; solo 5,5\% manifestó que todas las mujeres debían tener acceso al aborto provocado. De las mujeres entrevistadas, 19,0\% informó haberse sometido a un aborto provocado, de ellas 30,5\% declaró haber estado utilizando un método anticonceptivo moderno cuando tuvieron el embarazo no deseado.

La prevalencia general de abortos provocados fue de $16,3 \%$. Se encontraron tres factores asociados con este tipo de aborto: haber crecido en la ciudad (razón de posibilidades [odd ratio, OR] bifactorial: 1,60; OR de la regresión logística múltiple: 2,24), no haber parido (OR bifactorial: 1,60; OR de la regresión logística múltiple: 2,06) y haber tenido un embarazo no deseado (OR bifactorial: 2,09; OR de la regresión logística múltiple: 2,81 ). Una mayor proporción (aunque no estadísticamente significativa) de mujeres solteras de 15 a 24 años de edad informó haberse sometido a abortos provocados, en comparación con las mujeres casadas y de mayor edad.

La asociación encontrada entre haber tenido un aborto provocado y no tener hijos indica que se empleó el aborto para demorar la llegada del primer hijo, más que para espaciar el nacimiento de los hijos o para limitar el número total de hijos. Este resultado puede reflejar la necesidad de mejorar el acceso de las mujeres más jóvenes a los servicios de planificación familiar.

Aunque, según se analiza en el artículo, la TRA permitió llegar a una mejor estimación de la prevalencia de abortos provocados en México en comparación con otros métodos, los mejores resultados se obtuvieron al entrevistar mujeres con mayor nivel educacional y que habían crecido en ciudades. Se recomienda utilizar esta técnica para evaluar acciones $y$ actitudes que en un contexto dado puedan considerarse reprochables, sin embargo, se debe aplicar en un ambiente de privacidad y confianza. Se debe tener especial cautela al analizar la información obtenida mediante la TRA de personas analfabetas o de bajo nivel educacional. (Lara D, García SG, Ellertson C, Camlin C, Suárez J. The measure of induced abortion levels in Mexico using ranndom response technique. Sociol Meth Res. 2006;35(2):279-301.)

\section{Metaanálisis del desempeño de las pruebas de aglutinación directa y de la tira reactiva rK39 en el diagnóstico de la leishmaniasis visceral}

La leishmaniasis visceral es una enfermedad causada por parásitos del género Leishmania, que afecta anualmente a cerca de 500000 personas, principalmente en áreas rurales pobres del este de 
África, Asia meridional y América Latina. El diagnóstico certero y oportuno de esta enfermedad es de suma importancia, ya que puede ser mortal si no se trata a tiempo. El examen microscópico de muestras aspiradas del bazo es sensible y específico, pero requiere experiencia.

En los últimos años, dos pruebas serológicas han demostrado su utilidad en el diagnóstico de campo de la leishmaniasis visceral. La primera, la prueba de aglutinación directa, es semicuantitativa y legible a simple vista; está validada en diversas zonas endémicas y se utiliza ampliamente para el diagnóstico de la leishmaniasis visceral en varios países. La segunda y más reciente, una prueba inmunoenzimática basada en un antígeno recombinante compuesto por una secuencia repetitiva de 39 aminoácidos de Leishmania chagasi (rK39), tiene un formato más sencillo de tira reactiva y ofrece resultados cualitativos (positivo o negativo) en solo 20 minutos.

Para evaluar objetiva e independientemente el desempeño diagnóstico de ambas pruebas se realizó un metaanálisis con los estudios originales que evaluaban su capacidad diagnóstica en muestras de suero o sangre. Se tomaron en cuenta todos los artículos publicados entre enero de 1986 y diciembre de 2004, según la base de datos bibliográfica Medline. Dos revisores independientes valoraron los trabajos y sus resultados en cuanto al desempeño diagnóstico de las pruebas. Los desacuerdos se sometieron a un tercer revisor que solucionó los criterios discrepantes.

El metaanálisis de la sensibilidad y la especificidad de ambas pruebas se realizó mediante modelos de regresión logística. Para evaluar la heterogeneidad de los estudios se realizaron metaanálisis por separado en los subgrupos estratificados según la fase del estudio, el tamaño de la muestra, la calidad del estudio, la región geográfica, las especies de Leishmania circulantes, el tipo de antígeno empleado para la prueba de aglutinación directa, la marca comercial de la tira reactiva y los tipos de controles empleados.

En total se analizaron 30 estudios que evaluaron la prueba de aglutinación directa y 13, la tira reactiva rK39. Los estimados combinados de sensibilidad de la prueba de aglutinación directa y de la tira reactiva rK39 fueron 94,8\% (intervalo de confianza de 95\% [IC95\%]: 92,7\% a 96,4\%) y 93,9\% (IC95\%: 87,7\% a 97,1\%), respectivamente. La sensibilidad fue mayor y más homogénea en los estudios realizados en Asia meridional. La especificidad varió según el tipo de controles empleados. En los estudios de fase III, realizados en pacientes con signos clínicos de leishmaniasis, la especificidad de la prueba de aglutinación directa fue de 85,9\%
(IC95\%: 72,3\% a 93,4\%) y de la tira reactiva $\mathrm{rK} 39$, de 90,6\% (IC95\%: 66,8\% a 97,9\%).

Los resultados de este metaanálisis demostraron el buen desempeño de la tira reactiva rK39 y de la prueba de aglutinación directa en el diagnóstico de la leishmaniasis visceral. La sensibilidad general de ambas pruebas fue excelente.

El desarrollo de la prueba de aglutinación directa y, más recientemente, de la tira reactiva rK39 produjo una mejoría sustancial en el diagnóstico de campo de la leishmaniasis. Los resultados de este metaanálisis independiente apoyan el uso de estas pruebas diagnósticas. Como el desempeño de la prueba de aglutinación directa y de la tira reactiva rK39 son similares, la decisión de su uso podrá basarse en otros criterios, como el costo o la simplicidad. No obstante, la baja sensibilidad de la tira reactiva rK39 observada en Sudán y la menor especificidad de ambas pruebas serológicas en pacientes clínicamente sospechados de leishmaniasis visceral indican que se debe continuar investigando y desarrollando mejores herramientas de diagnóstico para esta enfermedad. (Chappuis F, Rijal S, Soto A, Menten J, Boelaert M. A meta-analysis of the diagnostic performance of the direct agglutination test and rK39 dipstick for visceral leishmaniasis. Br Med J. 2006;333:723.)

\section{Vigilancia de la infección de Trypanosoma cruzi en perros y gatos en una zona rural del noroeste de Argentina}

La enfermedad de Chagas, causada por el protozoo Trypanosoma cruzi, es la enfermedad parasitaria más importante en las Américas, con cerca de 17 millones de personas infectadas actualmente. Los esfuerzos por interrumpir la transmisión del parásito se han medido mediante paneles de seroprevalencia en humanos, pero esta práctica enfrenta limitaciones debido a consideraciones éticas. Se ha sugerido la posibilidad de utilizar a los perros como organismos centinela naturales de esta enfermedad, ya que presentan generalmente una prevalencia de infección por T. cruzi diez veces mayor que la de los niños.

Como parte de un estudio prospectivo a largo plazo dirigido a modelar la dinámica de transmisión de T. cruzi, se estudiaron los perros y gatos domésticos como centinelas naturales durante la fase de vigilancia para evaluar el efecto de la aplicación selectiva de insecticidas residuales en comunidades afectadas y la posible aparición de nuevos casos.

Para ello se evaluó periódicamente el nivel de infestación por chinches de todos los recintos habitados de las comunidades de Amamá, Trinidad, Mer- 